\title{
ENERGY EFFICIENT ROUTING IN CLUSTERED IOT WIRELESS SENSORS NETWORKS FOR QOS ENHANCEMENTS
}

\author{
A Sathesh \\ Department of Electronics and Communication Engineering, \\ Eritrea Institute of Technology, \\ Eritrea \\ E-mail:_sathesh4you@gmail.com
}

\begin{abstract}
The wireless sensor network that are randomly distributed with ability to communicate and to be communicated wirelessly appears to be vital part in the Internetwork of things to enable an autonomous communication between the visible commodities available in the tangible world. The process of the connection establishment between the WSNs and IOT for the purpose of information transmission is significant as it has to be reliable overcoming the challenges entitled in the wireless sensor networks. So the paper proposes an efficient clustering of WSN with a hierarchical proactive routing to have a transmission that affords within the limited battery availability and extended life of the network to have an improvised successful transmission rate, and diminished delay. The performance evaluation of the proposed system is conducted and compared with the previous methods to prove the energy efficiency and the QOS enhancements in terms of transmission rate and delay.
\end{abstract}

Keywords: Wireless sensor networks, Internet of things, Energy efficiency, Clustering- hierarchical proactive routing, QOS enhancements, Transmission rate and delay.

\section{Introduction}

The advancements in the technology and the innovations in the information sharing has attained a significance in the drastic changes caused in the day to day lives of the people, in the surroundings and the thing that are used. The emerging techniques of wireless communication has extended its information sharing capabilities even to remote, harsh and hostile environments seamlessly and are found to be economical. One such special case of adhoc network that communicates the gleaned information wirelessly is wireless sensor networks. These wireless network that designed for tacking, detection and prediction are packed with components that are essential in the monitoring, transmitting or receiving of the information and a processor along with the power storage to the equip its service. The random dispersed nature of this wireless sensor network enable them in establishing the process of information sharing to a wide area applications such as health monitoring, earth sensing, pollution monitoring ,fire/landslide detection, disaster monitoring, water quality, waste water monitoring, threat detection and even more reaching out to an enormous geographical arena with accuracy . This tracking and monitoring capabilities of the wireless sensor network has made it a prominent part of the internet of things which is an internetwork of internet enabled devices. 
Journal of ISMAC (2019)

Vol.01/ No. 01, June 2019, pp: 1-11

DOI: https://doi.org/10.36548/jismac.2019.1.001

ISSN: 2582-1369 (online)

The wireless sensors are preferred in the internetwork of things to provide with sovereign exchange of information between the tangible commodities that are embedded invisibly and identified uniquely with the help of a gateway and accessing points. The WSNs multifunctional abilities with reduced power consumption and cost makes them an optimized choice in the internet of things for a long term environmental tracking. The wireless motes that communicate without wires are to be routed perfectly with the internetwork of things to establish a proper to and fro communication. The nodes though available at low cost with least power consumption are decentralized and incur certain natures such as high mobility deviation, storage power limitations, limited band width and sudden link failures that make the routing provision to be challenging with the traditional available methods. The traditional flat routing technologies with collision overhead, latency, complex routing strategies without synchronization and link stability are side stepped with the enabling hierarchical routing that ensures in having a routing with the ability of managing the information sharing with the limited battery power and the bandwidth available, with a stable connection establishment..

The proposed method uses a hierarchical proactive routing based on clustering to support in the establishment of stable connections that ensure a limited battery usage with negligible packet losses and reduced overhead causing an increase in the successful transmission with diminished delay. The paper with the rest of its portion is organized with 2 the related works, 3 proposed work, 4 Results and evaluation and 5 Conclusion.

\section{Related works}

Wang et al [1] to have an improvised spectral efficiency in the fields of medical, industry and scientific research by the exploitation of the cross layer technology in the process of transmission for having an improvisation in the packet delivery ratio and reduction in the delay incurred in the delivery. Lin et al [2] the method provides with the formation of the virtual cluster head to elude the performance degradation caused on the cluster head that turns out to be damaged during the transmission. As the cluster head is the acts a server in gathering the information from the sensor motes and transmitting it to the base station any damages incurred in them would affect the gathering as well as the transmission process so the method relies on virtualization of cluster head to elude the failures of the cluster head. Gunatilaka et al [3] uses a reliable, adaptive and effective plane of centralized network management to have an improvisation in real time performance, route diversity and channel reuse for maintain a stable route for having continuous transmission on the same channel with increased reliability. Hezaveh et al [4] the battery power limitations of the wireless sensor networks causes sudden node failures causing irregularities in the transmission. The fault tolerant and energy efficient routing enables life time prolongation of the nodes by evading the node overlap causing the powerful nodes to sleep and using their energy for the cluster nodes that are depleted of energy. Pai et al [5] gives the performance on the topological analysis of the IPV6 over low power wireless personal area networks for the wireless sensor networks along with IOT. This enables in perfect regrouping on the occurrence of 
Journal of ISMAC (2019)

Vol.01/ No. 01, June 2019, pp: 1-11

DOI: https://doi.org/10.36548/jismac.2019.1.001

ISSN: 2582-1369 (online)

node failure. Alarifi et al [6] involves the clustering based on Q learning affords an improved intra and inter communication between the clusters by the selection of adaptive forwarder and header to have a reduced overhead with improvement in network lifetime causing stable connections. Saranya, et al [7] is a modified low energy adaptive clustering and a multi-hop method for routing to have an improved network life time by the selection of the cluster head based on the residual energy, the distance and its data over head. Zheng et al [8] is a network stability clustering protocol for purposes of having an energy efficient routing with stable connection establishments. Kwon, et al [9] uses a cluster head selection with reduced complexity based on the capacity of the clique and the members of the cluster based on the characteristics of the cluster head using a fuzzy interference system to have a delay-less, energy efficient transmission in the IOT-WSN. Li, et al [10], uses a wireless power transmission for satisfying the power requirement of the IOT connected devices and uses machine learning algorithm in combination with k- means algorithm to have a proper header and broadcaster to have an efficient routing with diminished packet losses. Farman et al [11] the zone head selection based on the its distance, energy level and the number of times it has been a zone head to have optimal selection on the header that directly impacts on the network life time and stability. Cui et al [12] uses a weight harmonic centroid algorithm integrated with the LEACH to have a double stage selection of head and member to save energy. Kaiwartya et al [13] uses a non-dominant sorting based genetic algorithm with computation, mutation and cross over for developing virtual WSNs for the purpose of replacing the wireless sensor networks that faces failures during transmission.

\section{Proposed Scheme using the Clustered architecture and proactive way of transmission}

The distributed, decentralized and highly mobile wireless sensor networks are framed into clusters in the proposed method to have an improved path realization, with lowest path delays and overheads with enhanced facilities for information gleaning and reduced node failures including scalability. The limitations in the battery being the main cause for node failures the proposed clustering method for routing is to reduce the processing's thereby causing reduction in the usage of energy to extend the life time of the clique and enhancing the transmission rate.

The cluster framing that is put forward in this energy efficient routing with QOS enhancements follows a hierarchical clustering that are acquiesce in bringing to gather the nodes with similar features into a troop with certain surmise that are as follows, the nodes indulged in sensing are familiar with their position related to the accessing points, and are homogenous, the signal to noise ratio of the channel are regulated so as to help in affording the energy for signal transmission, and the position of the base point being well scheduled with sensing nodes at locale. 
Journal of ISMAC (2019)

Vol.01/ No. 01, June 2019, pp: 1-11

DOI: https://doi.org/10.36548/jismac.2019.1.001

ISSN: 2582-1369 (online)

The method used in the proposed is to have a reduced energy consumption by reducing the number of proceedings in the route establishment. So the proffered method chooses to move with proactive way of path finding so as to reduce the losses incurred in form of delay, overhead and increased energy usage due to the extra signaling messages in the routing process that is reactive.

\subsection{Cluster Framing}

The cluster framing is proceeded by selecting a head for the grouped nodes followed by enrolling the other nodes into them as members of the group. The member enrollment into a particular group is organized by initiating a requisition from the head of the group to other data points that does not hold the position of head and fall within the imparting realm of the head of the group that is given in equation (1)

$$
\mathrm{I}_{\mathrm{R}}=\sqrt{\mathrm{P}_{\mathrm{s}}}\left(\frac{\text { Total }_{\text {area }}}{P_{b} \Pi \text { Total }}\right)
$$

Where is the imparting realm $\mathrm{I}_{\mathrm{R}}, \mathrm{P}_{\mathrm{s}}$ is the tunable variable of the device, Total area $_{\text {is }}$ the complete area of the

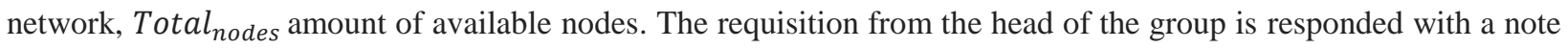
of interest in being enrolled to the group. The node with inquisitiveness is computed for its energy level, and its distance from the neighboring nodes. The data points with its initial energy lesser than the energy level of the head of the group is accumulated into the group after calculating with the degree of the head ( $\left.\mathrm{Head}_{\text {degree}}\right)$ that is given by the equation (2)

$$
\text { Head }_{\text {degree }}=\frac{\text { Group }_{\text {nodes }}}{\text { Nodes }_{\text {realm }}}-1
$$

Where the Group $_{\text {nodes }}$ total number of nodes in the group, Nodes realm $_{\text {is }}$ is the number of nodes within the realm of the head. The Head ${ }_{\text {degree }}$ less than zero intimates the space availability for adding more data points to the group and Head $_{\text {degree }}$ equal to zero represents the adequate amount of nodes added in the group and the lack of space for further enrollment. The head of the group that initiates the framing of the group possess a superior characteristics in terms of energy and distance in comparison to the other nodes that are present in the group. The randomly dispersed nodes are enrolled in either any one of the cluster head under the conditions being met. As mentioned above the data points are battery powered and are liable to lose its energy whenever they are used for transmission, so the cluster head on losing its energy becomes dead making its position vacant for the next head. The next head is locally elected within the group without much energy wastage considering the vestigial energy of the nodes within the realm of the group given by equation (3) 
Journal of ISMAC (2019)

Vol.01/ No. 01, June 2019, pp: 1-11

DOI: https://doi.org/10.36548/jismac.2019.1.001

ISSN: 2582-1369 (online)

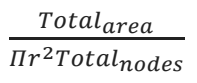

And its distance limited to two hops, by which the other nodes in direct contact could reach the head.

\subsection{Proactive routing for energy efficiency}

The proactive routing followed by the proffered method enables in having the complete information of the data points, the possible ways that can be routed and the regular updation of the clique to all its sensing nodes. So this ensures the network with the shortest route availability for extending the transferal without any need for the excess energy wastage on the path finding process. The quick option on the already available route and session establishment enables the network in having numerous alternate paths to select with, on the failure of the particular node. The regular updation process keep the network updated of the node and link failure and the possible rerouting to be carried out at a very high speed.

The optimized routing path for the transferal is to be selected considering the number of hops that are required for the conveyance. The minimum number of hops are decided to make use of the shortest path for the transferal so the nodes covering the destination with the minimum amount of hops are preferred to be the optimized transferal path.

The information from the sensing nodes are transmitted to the cluster head and from the cluster head to the base station. The sensing nodes which under the direct locale of the cluster head commune directly whereas the nodes beyond the vicinity of the cluster head use an intermediate node for communing. The intermediate sensing node with minimum number of strides are selected as the optimized intermediates for communicating with the head forming a temporary set up of link to extend communication from the cluster to base station.

The power consumption assuming that all the nodes where in vicinity with the head is given by equation (4)

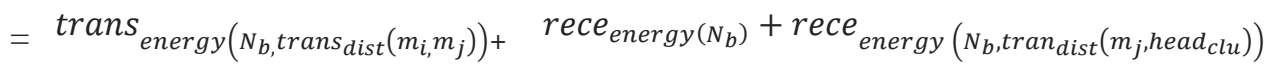

With the total power consumption of the path extended for transmission is given by equation (5)

$=N_{b}$, trans $_{\text {dist }\left(m_{i}, \text { head }_{\text {clu }}\right)^{\wedge} 2}$ 
Journal of ISMAC (2019)

Vol.01/ No. 01, June 2019, pp: 1-11

ISSN: 2582-1369 (online)

DOI: https://doi.org/10.36548/jismac.2019.1.001

The method proposed prefers a cost -effective way for forming a path based on the clustered architecture, the paths available beforehand eludes the unwanted power consumption caused on the signaling used with the processing of the path extension. Thus reducing the amount of proceedings required in establishing the path results in the reduction in the power consumption, the decision made locally for carrying out the rerouting and re-election of the head also causes further reduction in power consumption thus enabling a continuous transferal afforded by the limited battery available on the sensing nodes with negligible failure rates

\section{Result Analysis}

The proffered process with the conception of energy conservation and to have extension in the life span of the network is evaluated using the network simulator, with the number of sensing nodes varying from hundred to five hundred. The evaluation further is compared with the route processing's that is reactive to gain knowledge regarding the improvisation attained in the proposed process in terms of QOS enhancements. The parameter values are listed below in the Table 1.

Table 1

\begin{tabular}{|c|c|}
\hline Parameters & Value \\
\hline simulator & NS-2 \\
\hline Number of automobiles & $100-500$ \\
\hline Initial energy & 100 joules \\
\hline Packet size & $1024 \mathrm{bits}$ \\
\hline Allotted area & $2500 * 40 \mathrm{~m}$ \\
\hline Packet data rate & I packet /second \\
\hline
\end{tabular}

\section{a) Delay less transmission}

The proposed routing proceedings that is proactive reduces the delay in the process of route entrenchment, as the routing are available beforehand. Whenever the source is ready with the information to be transmitted, immediately the optimized path is achieved by just deciding with the previously available path that is enriched with the sensing nodes that cover the destination at minimum hops. The rerouting of the path and re-election of the head is also less time consuming due to the regular update of the information's regarding the sensing data points that is carried out in this proactive process. So the transmission from the source to destination does not consume much time. 


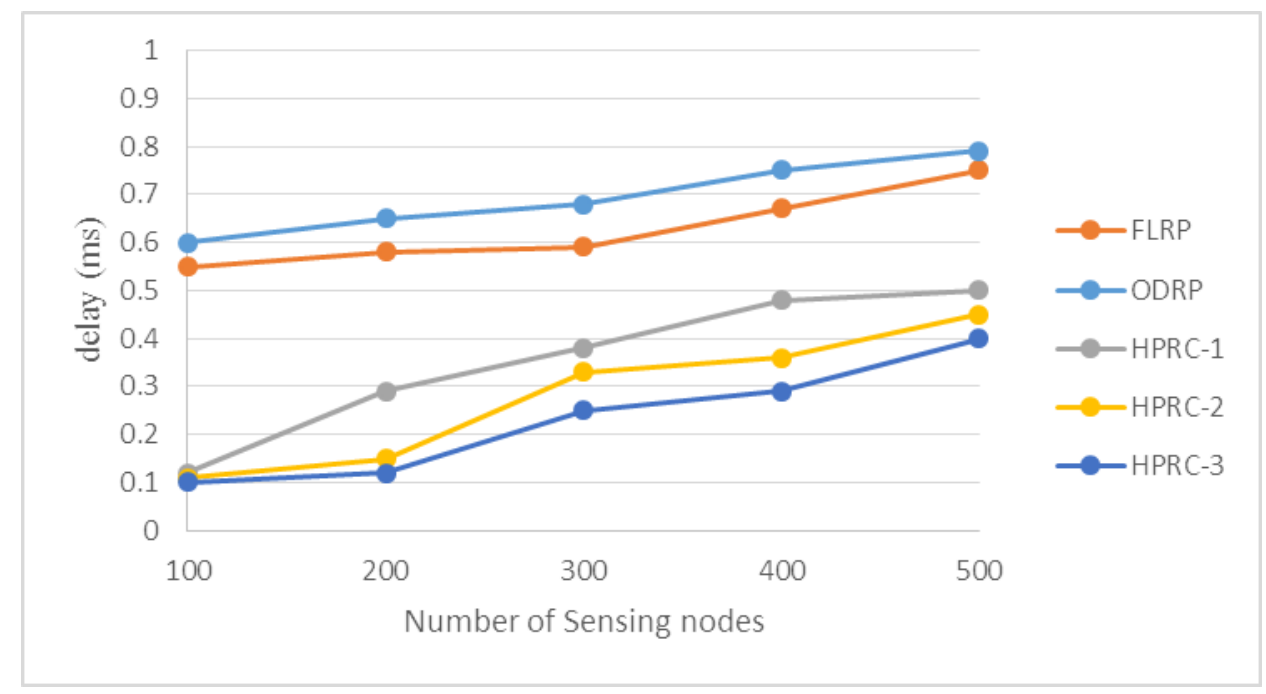

Fig 1 Delay Calculation

The delay for the proposed system is given by finding the time difference between the transmission and the reception of the information. The delay curve in the FIG 1shows the delay attained by the proposed system at different simulation set up with number of sensing nodes varying from 100-500. The negligible delay incurred by the proffered method show the limited bandwidth usage of the process without overhead. The results when compared to the prevailing reactive methods evidences the proposed method is deft with negligible delay.

\section{b) Energy utilization}

The preeminent objective of the proposed process is the minimum energy utilization which is achieved by routing discovery enabled by the limited signaling and the re-routing and the re-selection process that are decided locally. The energy utilization graph shown in Fig 2 shows the reduced energy usage of the proffered method. The lessened energy usage evidences the extended life time of the network and the increased number of alive nodes. The comparison with prevailing methods proves the proficiency of the proposed method in terms of energy utilization. The energy utilization is evaluated is given by the equation (7) 
Journal of ISMAC (2019)

Vol.01/ No. 01, June 2019, pp: 1-11

ISSN: 2582-1369 (online)

DOI: https://doi.org/10.36548/jismac.2019.1.001

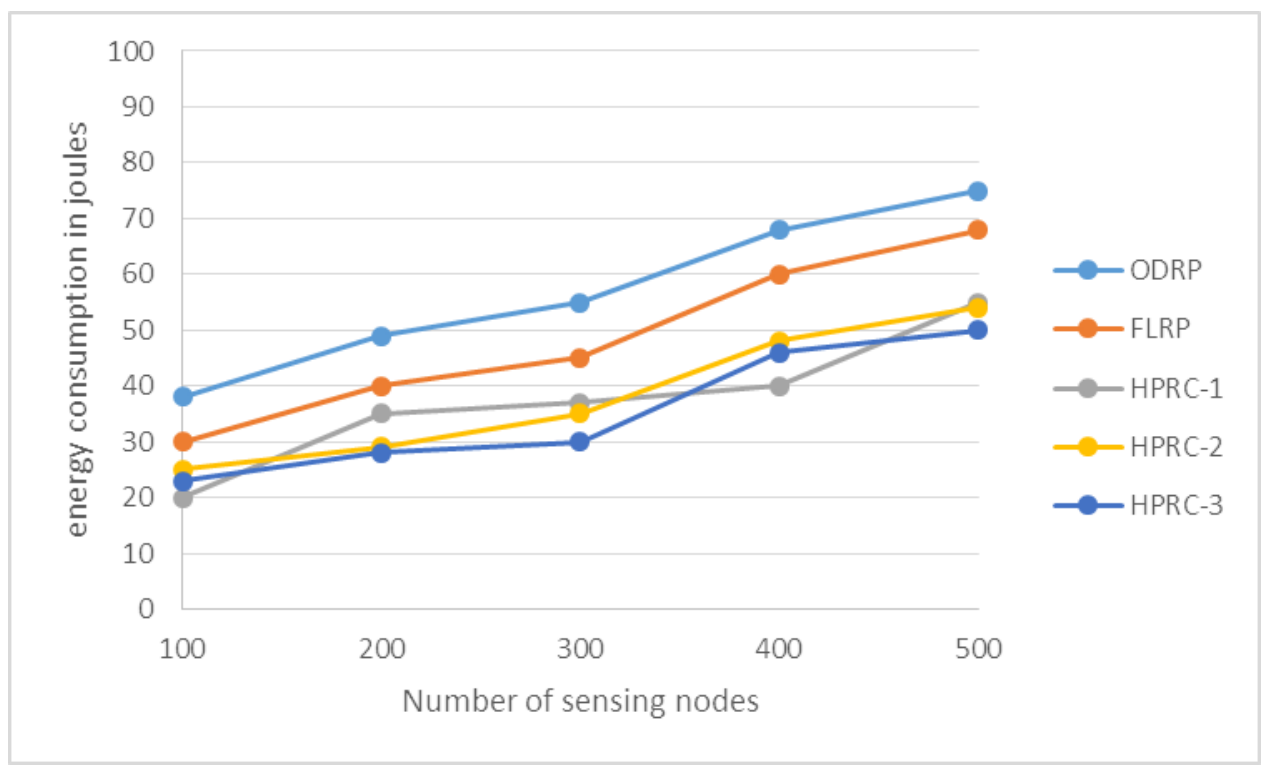

Energy utilization $=$ trans $_{\text {energy }} * N_{b}+$ rece $_{\text {enerrgy }} * N_{b}$

Fig 2 Energy Utilization

\section{c) Throughput}


Journal of ISMAC (2019)

Vol.01/ No. 01, June 2019, pp: 1-11

DOI: https://doi.org/10.36548/jismac.2019.1.001

The successful transmission for the proposed proceeding is attained by the number of successful reception for the number of information transferred. The successful transmission helps in measuring the total number of successful packet deliveries, the losses incurred with regard to the packets and the failure addressed due to the death of the sensing nodes.

$$
\text { Throughput }=\frac{\text { No.of successful reception }}{\text { No.of transmissions }}
$$

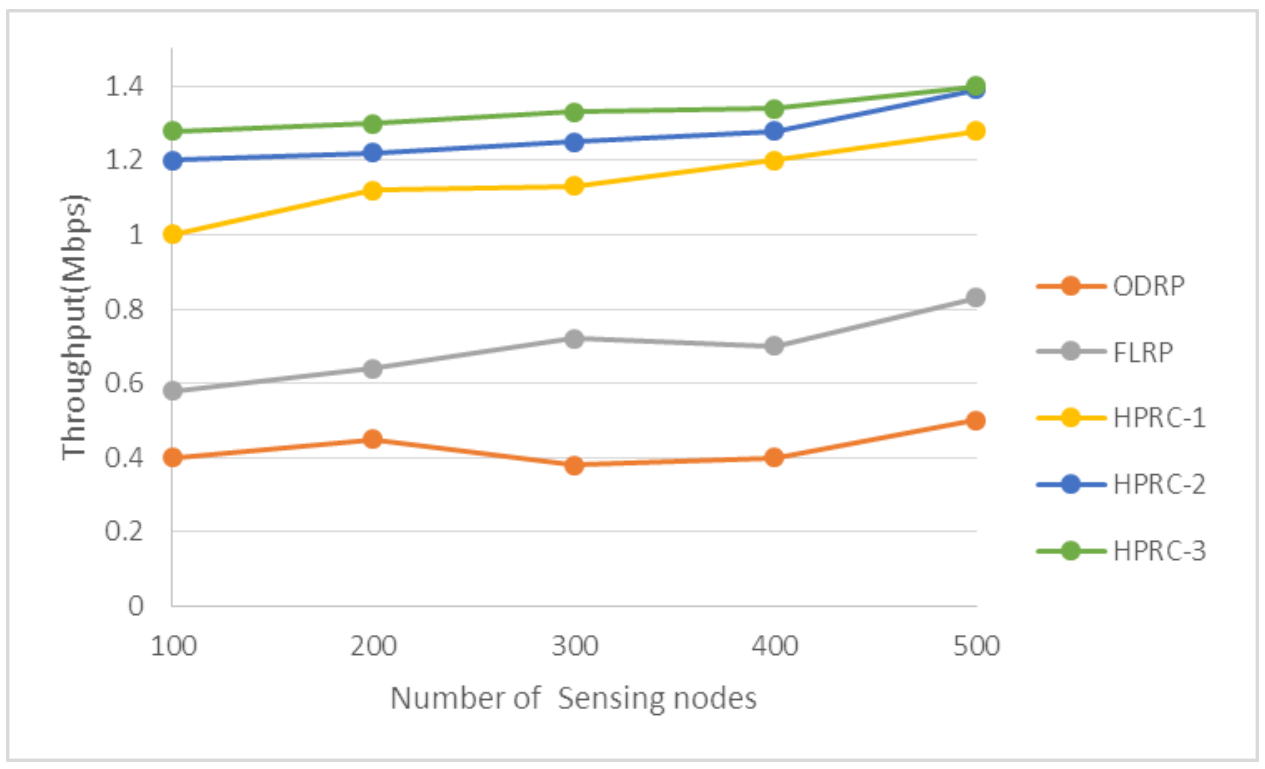

Fig 3 Throughput

The equation (7) shows the throughput calculation of the proposed. The Fig 3 give the throughput calculation for presence of the number of nodes that varies from 100 to 500. The different simulation setups and the comparison with the preexisting methods show that the proposed method is deft with enhanced throughput.

\section{Conclusion}

The paper proposes a hierarchical proactive routing with clustering by enabling a hierarchical cluster framing with nodes of similar features (homogenous). The process enabled in the routing are limited due to the availability of the route that are ready made using the proactive method that is dissimilar to on demand routing procedures. These provisions of already available routing and the localized settlements regarding the re-establishing of the path and reelection of the head and arbitrary path establishment through the cluster to the base station make the proposed 
Journal of ISMAC (2019)

Vol.01/ No. 01, June 2019, pp: 1-11

DOI: https://doi.org/10.36548/jismac.2019.1.001

ISSN: 2582-1369 (online)

method deft with regard to energy utilization, with enhanced quality of service achieving improved through put and lessened delay. The performance evaluation and comparison with the prevailing methodologies proves the adeptness of the proposed process.

\section{References}

[1] Wang, Wei, Tiantian Xie, Xin Liu, Yao Yao, and Ting Zhu. "ECT: Exploiting Cross-Technology Transmission for Reducing Packet Delivery Delay in IoT Networks." ACM Transactions on Sensor Networks (TOSN) 15, no. 2 (2019): 20.

[2] Lin, Jenn-Wei, Pethuru Raj Chelliah, Meng-Chieh Hsu, and Jia-Xin Hou. "Efficient Fault-Tolerant Routing in IoT Wireless Sensor Networks Based on Bipartite-Flow Graph Modeling." IEEE Access 7 (2019): 14022-14034.

[3] Gunatilaka, Dolvara. "High Performance Wireless Sensor-Actuator Networks for Industrial Internet of Things." PhD diss., Washington University in St. Louis, 2019.

[4] Hezaveh, Maryam, Zahra Shirmohammdi, Nezam Rohbani, and Seyed Ghassem Miremadi. "A faulttolerant and energy-aware mechanism for cluster-based routing algorithm of WSNs." In 2015 IFIP/IEEE International Symposium on Integrated Network Management (IM), pp. 659-664. IEEE, 2015.

[5] Pai, Vasudeva, and Udaya Kumar K. Shenoy. "6LowPan-Performance Analysis on Low Power Networks." In International Conference on Computer Networks and Communication Technologies, pp. 145-156. Springer, Singapore, 2019.

[6] Alarifi, Abdulaziz, and Amr Tolba. "Optimizing the network energy of cloud assisted internet of things by using the adaptive neural learning approach in wireless sensor networks." Computers in Industry 106 (2019): 133-141.

[7] Saranya, V., S. Shankar, and G. R. Kanagachidambaresan. "Energy efficient clustering scheme (EECS) for wireless sensor network with mobile sink." Wireless Personal Communications 100, no. 4 (2018): 15531567.

[8] Zheng, Meng, Si Chen, Wei Liang, and Min Song. "NSAC: A Novel Clustering Protocol in Cognitive Radio Sensor Networks for Internet of Things." IEEE Internet of Things Journal (2019).

[9] Kwon, Jung-Hyok, Minki Cha, Sol-Bee Lee, and Eui-Jik Kim. "Variable-categorized clustering algorithm using fuzzy logic for Internet of things local networks." Multimedia Tools and Applications 78, no. 3 (2019): 2963-2982.

[10]Li, Lixin, Yang Xu, Zihe Zhang, Jiaying Yin, Wei Chen, and Zhu Han. "A prediction-based charging policy and interference mitigation approach in the wireless powered Internet of Things." IEEE Journal on Selected Areas in Communications37, no. 2 (2019): 439-451. 
[11]Farman, Haleem, Bilal Jan, Huma Javed, Naveed Ahmad, Javed Iqbal, Muhammad Arshad, and Shaukat Ali. "Multi-criteria based zone head selection in Internet of Things based wireless sensor networks." Future Generation Computer Systems 87 (2018): 364-371.

[12] Cui, Zhihua, Yang Cao, Xingjuan Cai, Jianghui Cai, and Jinjun Chen. "Optimal LEACH protocol with modified bat algorithm for big data sensing systems in Internet of Things." Journal of Parallel and Distributed Computing (2018).

[13] Kaiwartya, Omprakash, Abdul Hanan Abdullah, Yue Cao, Jaime Lloret, Sushil Kumar, Rajiv Ratn Shah, Mukesh Prasad, and Shiv Prakash. "Virtualization in wireless sensor networks: Fault tolerant embedding for internet of things." IEEE Internet of Things Journal 5, no. 2 (2018): 571-580. 